\title{
A histopathologic study of fatal paediatric cerebral malaria caused by mixed Plasmodium falciparum/Plasmodium vivax infections
}

\author{
Laurens Manning $^{1 \dagger}$, Anna Rosanas-Urgell ${ }^{2 \dagger}$, Moses Laman ${ }^{1,2}$, Henry Edoni ${ }^{2}$, Catriona McLean ${ }^{3}$, Ivo Mueller ${ }^{2,4,5}$, \\ Peter Siba ${ }^{2}$ and Timothy ME Davis ${ }^{{ }^{*}}$
}

\begin{abstract}
Microvascular sequestration of Plasmodium falciparum underlies cerebral malaria. Despite suggestive ex vivo evidence, this phenomenon has not been convincingly demonstrated in coma complicating Plasmodium vivax malaria. Severely-ill Papua New Guinean children with mixed P. falciparum/P. vivax infections are more likely to develop cerebral malaria and die than those with $P$. falciparum alone, possibly reflecting $P$. vivax sequestration. Nested PCR was performed on post mortem brain tissue from three such children dying from cerebral malaria due to mixed-species infections. No $P$. vivax DNA was detected. These findings do not support the hypothesis that $P$. vivax sequestration occurs in human brain.
\end{abstract}

Keywords: Plasmodium vivax, Post mortem biopsy, PCR, Cerebral malaria, Children

\section{Background}

Plasmodium vivax accounts for nearly half of all malaria infections and is now recognized as a cause of complications and death [1]. Cerebral malaria due to P. vivax, although rare, has been reported from Indonesia, India and Papua New Guinea (PNG) [2-4]. In patients with altered consciousness due to Plasmodium falciparum, late stage parasites (trophozoites and schizonts) can be found sequestered within the brain microvasculature. There is no convincing evidence of a similar phenomenon in post mortem brain specimens from patients with $P$. vivax, but available data are few and from studies in which interpretation of the histopathologic features is confounded by issues such as the possibility of unrecognized mixed-species infections [5].

Animal and in vivo functional studies reveal that $P$. vivax sequesters preferentially in the pulmonary and splenic microvasculature [5], consistent with clinical studies in which pulmonary manifestations and severe anaemia occur relatively frequently $[2,6]$. Ex vivo studies

\footnotetext{
*Correspondence: tdavis@cyllene.uwa.edu.au

† Contributed equally

'School of Medicine and Pharmacology, University of Western Australia,

Fremantle Hospital, PO Box 480, Fremantle 6959, Western Australia, Australia Full list of author information is available at the end of the article
}

have shown cytoadherence of $P$. vivax to chondroitin sulphate A, erythrocyte rosetting and endothelial adherence $[5,7,8]$, albeit at lower avidity than $P$. falciparum. Nevertheless, these observations raise the possibility that cerebral microvascular sequestration may underlie cerebral malaria due to $P$. vivax.

Apart from research settings [9], autopsy studies of patients dying from malaria are rarely performed. In the case of fatal cerebral vivax malaria, there have been no published post mortem studies for $>60$ years [5]. The results of a prospective observational study of severely ill PNG children with malaria were reported recently [2]. Those with mixed $P$. falciparum/P. vivax infections were more likely to have impaired consciousness and to die than those infected with $P$. falciparum alone. In the present study, histopathologic and molecular studies were performed using brain tissue from three of the fatal cases in this series [2] in order to examine the hypothesis that cerebral sequestration of $P$. vivax contributed to their presenting neurological features and outcome.

Details of the main prospective observational study from which the present cases are drawn have been published [2]. In brief, all children aged 0.5-10 years admitted to Modilon Hospital, Madang Province, between October 2006 and December 2009 were assessed for recruitment 
to an observational study of severe paediatric illness. Inclusion criteria reflected the World Health Organization (WHO) definition of severe malarial illness. All children were screened for malaria by both blood film microscopy and subsequent diagnostic and Plasmodium species confirmation by nested polymerase chain reaction (nPCR) [10]. In-patient management was co-ordinated by attending ward clinicians under PNG national guidelines.

Cerebral malaria was defined as a Blantyre Coma Score $\leq 2$ with Plasmodium DNA detected in peripheral blood by nPCR [2], irrespective of the presence and/or species of asexual parasites by microscopy. A mixed $P$. falciparum/P. vivax infection was defined as the presence of both species DNA by nPCR, regardless of species or parasitaemia detected microscopically. Post mortem brain tissue was obtained using a bone marrow biopsy needle via a supra-orbital approach [9]. Small fragments of brain tissue were placed between two slides, gently squashed, stained with $2.5 \%$ Giemsa for one hour before microscopic examination. Additional pieces of brain tissue were stored in formalin and liquid nitrogen. Formalin-fixed tissue was embedded in paraffin, sectioned and stained with haematoxylin and eosin (H\&E), Gram's, Giemsa and silver stain prior to microscopy which was performed by a specialist neuropathologist $(\mathrm{CM})$ in a pathology laboratory accredited by the Australian National Association of Testing Authorities.

Initial Plasmodium species identification was performed on peripheral blood by nPCR [10] after parasite DNA extraction (QIAamp 96 DNA Blood Mini Kit, QIAGEN, Valencia, CA). Positive samples for mixed P. falciparum/ $P$. vivax infection were quantified by $\mathrm{PCR}$ (qPCR) using validated methods [11]. Brain tissue stored in liquid nitrogen or formalin was homogenized before DNA extraction (DNeasy Blood \& Tissue Kit, QIAGEN, Valencia, CA, USA) prior to $\mathrm{nPCR}$.

\section{Case presentations}

Three children of 340 with severe malaria died from cerebral malaria and underwent post mortem brain biopsy. No brain biopsy material was available for the other three children in the series as a whole who died. All three of the present children had a mixed $P$. falciparum/P. vivax infection detected from peripheral blood by nPCR. Their admission clinical and laboratory features are summarized in Table 1.

\section{Case 1}

This child presented in deep coma without other features of severe malaria and died within hours of admission despite prompt anti-malarial therapy and supportive treatment. P. falciparum was present on blood smear and nPCR, but $P$. vivax was not identified by microscopy and only at low levels ( $>40$ cycles) by qPCR. The qPCR data represent a $P$. falciparum and $P$. vivax parasitaemia of 10,000 and $<10$ parasites $/ \mu \mathrm{L}$, respectively [11]. Microscopy of brain tissue revealed occasional $P$. falciparum parasites and no identifiable $P$. vivax within the cerebral vasculature (Figure 1). No inflammation, haemorrhage or necrosis was observed in the adjacent brain parenchyma. Plasmodium falciparum but not $P$. vivax was present in brain by $\mathrm{nPCR}$.

\section{Case 2}

This patient presented with cerebral malaria, metabolic acidosis, hyperlactataemia and severe anaemia. This child died within hours of admission despite prompt anti-malarial therapy and supportive treatment. P. falciparum and $P$. vivax in peripheral blood were identified by microscopy, nPCR and qPCR. Using qPCR, P. falciparum and P. vivax DNA concentrations indicated equivalent parasitaemia of 50,000 and $500 / \mu \mathrm{L}$, respectively. Plasmodium falciparum, but not $P$. vivax, was present in brain by nPCR. Malaria pigment and numerous $P$. falciparum trophozoites and schizonts were visible within the microvasculature on histologic examination (Figure 1). Other than subtle focal upregulation of microglial cells, there were no abnormalities observed in the adjacent brain parenchyma.

\section{Case 3}

This child presented deeply comatose. Seven days before admission, he attended a local clinic with headache and fever and was prescribed amodiaquine, sulphadoxine and pyrimethamine. He did not improve and was given intramuscular artemether five days later. On admission to Modilon Hospital, blood film microscopy and a rapid diagnostic test for malaria were negative, but both species were subsequently detected by nPCR of peripheral blood and at low levels by qPCR (10-100 parasites/ $\mu \mathrm{L})$. At lumbar puncture, there were 5 leucocytes/ $\mu \mathrm{L}$ (100\% lymphocytes) in the cerebrospinal fluid (CSF) and the CSF protein concentration was $3 \mathrm{~g} / \mathrm{L}$. Blood cultures were negative. He did not regain consciousness and died four days later despite intravenous dextrose/saline and antibiotics, and intramuscular artemether.

Neither P. falciparum nor P. vivax was present in the brain of Patient 3 by nPCR. Further molecular testing of CSF and brain tissue for arbo-, Henipah, Entero- and Herpes viruses did not reveal an alternative diagnosis. Histopathologic examination of brain tissue showed infarction of cortical tissue with acute chromatolysis of neurons and early infiltration by neutrophils from adjacent vessels at the edge of the infarction (Figure 1). Adjacent white matter was unaffected and cerebral vessels appeared normal. No lymphocytes, histiocytes, granulomata, malarial parasites, fungal hyphae, bacteria or emboli were seen. The changes were interpreted as cortical infarction of at least 24 hours duration without a definitive aetiology. 
Table 1 Clinical and laboratory features of children dying from cerebral malaria due to mixed Plasmodium falciparum/ Plasmodium vivax infections

\begin{tabular}{|c|c|c|c|}
\hline & Patient 1 & Patient 2 & Patient 3 \\
\hline Age (months) & 63 & 36 & 94 \\
\hline Sex & Female & Female & Male \\
\hline Blantyre coma score on admission & 0 & 2 & 1 \\
\hline Blood lactate $(\mathrm{mmol} / \mathrm{L})$ & 1.6 & 6.4 & 1.0 \\
\hline Plasma bicarbonate (mmol/L) & 18 & 10.2 & 22.3 \\
\hline Blood glucose (mmol/L) & 18.4 & 3.2 & 7.0 \\
\hline Haemoglobin (g/L) & 69 & 39 & 111 \\
\hline \multicolumn{4}{|c|}{ Peripheral parasitaemia $(/ \mu \mathrm{L})$ by light microscopy: } \\
\hline P. falciparum & 154,000 & 104,000 & 0 \\
\hline P. vivax & 0 & 80 & 0 \\
\hline Plasmodium species by nPCR (blood) & Mixed species & Mixed species & Mixed species \\
\hline \multicolumn{4}{|c|}{ Peripheral parasitaemia by qPCR (number of cycles): } \\
\hline P. falciparum & 26.3 & 23.1 & 37.2 \\
\hline P. vivax & 41.0 & 29.2 & 35.8 \\
\hline Plasmodium species by nPCR (brain) & P. falciparum & P. falciparum & Negative \\
\hline
\end{tabular}

$\mathrm{nPCR}$, nested polymerase chain reaction [10]; qPCR, quantitative polymerase chain reaction [11]

\section{Conclusions}

The present case series provides the first detailed histopathologic and molecular study of the potential role of $P$. vivax in the pathogenesis of coma as a manifestation of severe malaria. There was no evidence for cerebral sequestration of $P$. vivax in any of the three cases despite microscopic and/or molecular confirmation that this parasite was present in peripheral blood at presentation. Thus, despite ex vivo studies suggesting that cytoadherence of $P$. vivax might contribute to complications $[5,7,8]$, the present data do not support the hypothesis that this phenomenon occurs in the human brain.

Patient 1 was diagnosed and treated as a case of cerebral malaria due to a P. falciparum mono-infection. By implication from the peripheral blood microscopy and qPCR data, this young girl's total $P$. vivax parasite burden was relatively low and the negative cerebral histopathology and nPCR could have reflected this. Mature P. falciparum forms in the brain in this patient were scarce compared to the peripheral parasitaemia $(154,000 / \mu \mathrm{L})$. This suggests that she had a synchronous infection with either maturing parasite forms starting to cytoadhere, or (less likely in view of the lack of intravascular malaria pigment) maturation and rupture of schizonts just before presentation. Whether a similar phenomenon might operate in the case of P. vivax is unknown. In a typical patient with $P$. falciparum, about half of the infected erythrocytes are sequestered [12]. It is possible that the dynamics of putative $P$. vivax sequestration with less avid ligand binding [7] are different to P. falciparum in that the majority of parasites remain in the peripheral blood. This could explain the weakly positive PCR in blood and negative PCR in the brain of this patient if $P$. vivax cytoadherence were possible.

Patient 2 provides the strongest evidence against $P$. vivax cerebral cytoadherence. This child had a mixedspecies infection identifiable by peripheral blood microscopy but nPCR of brain tissue was only positive for P. falciparum. The difference in peripheral blood $P$. vivax density observed by qPCR compared to microscopy (500 vs $80 / \mu \mathrm{L}$, respectively) is likely to reflect the fact that even very experienced microscopists often overlook a second infecting species in mixed infections [13]. Brain histology in this patient showed an abundance of $P$. falciparum and its greater avidity for endothelial ligands under both static and flow conditions [7] may have competitively inhibited $P$. vivax cytoadherence. However, the distribution of $P$. falciparum sequestration varies significantly between cerebral vessels in the same patient [14] and, in any case, the absence of $P$. vivax by nPCR implies that this parasite had no direct role in causing deep coma.

Patient 3 was included in the present series because of the positive peripheral blood PCR results. In studies of $P$. falciparum, mature parasite forms are no longer visible in brain biopsy specimens three days after anti-malarial treatment [14] and this patient died seven days after intramuscular artemether. It is, therefore, not surprising that brain nPCR was negative for P. falciparum and, even if cerebral $P$. vivax sequestration had contributed to coma, that brain $\mathrm{nPCR}$ was also negative for $P$. vivax. Ischemia and infarction can occur in cerebral malaria caused by P. falciparum in children [15]. It is likely that histologic appearances in this patient, coupled with prior anti-malarial treatment, low-grade parasitaemia detected by PCR and 


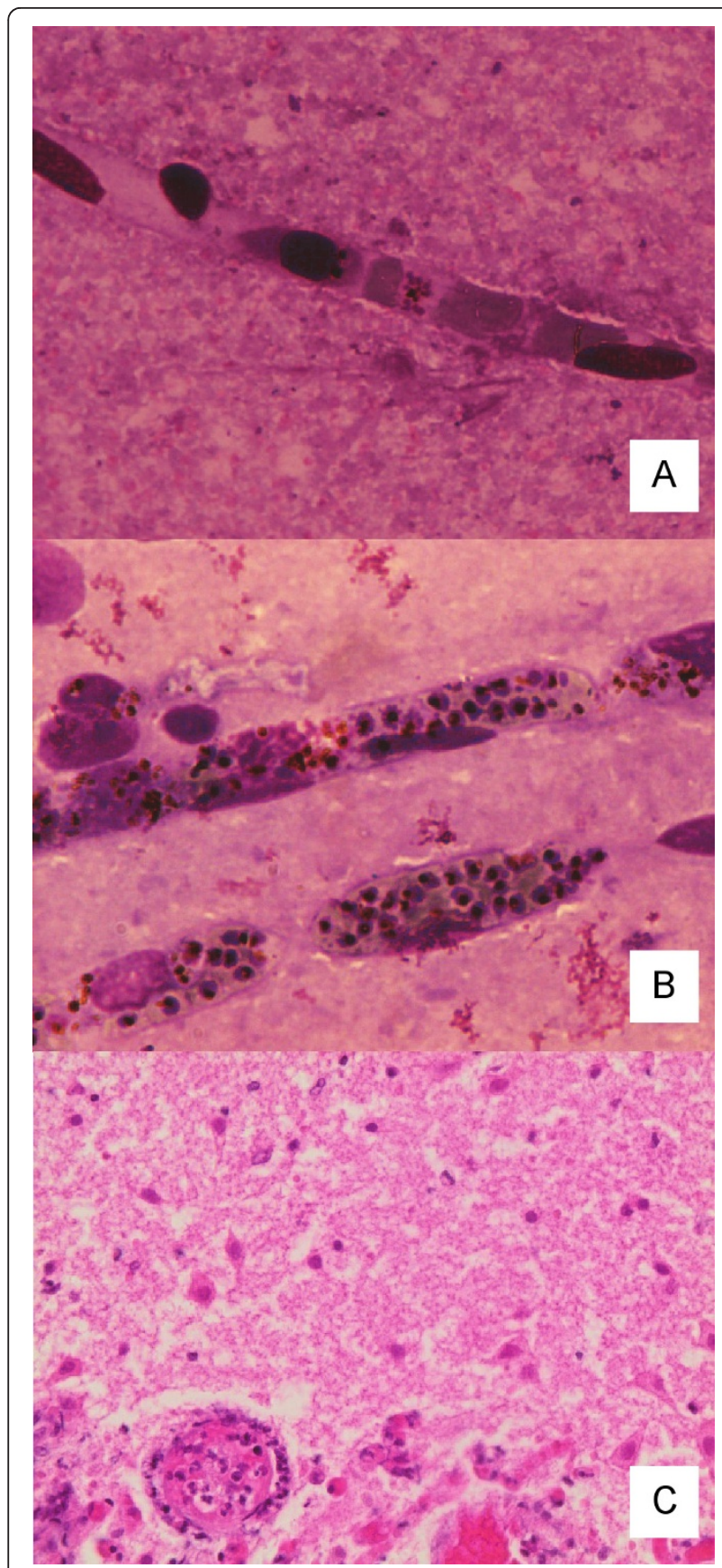

Figure 1 Light microscopy of brain tissue obtained from children dying from cerebral malaria due to mixed

Plasmodium falciparum/Plasmodium vivax infections. In panel $\mathbf{A}$ (Patient 1), occasional mature forms of $P$. falciparum were seen (Giemsa, magnification $\times 400$ ). In panel B (Patient 2), malaria pigment and numerous $P$. falciparum trophozoites and schizonts are visible within the microvasculature (Giemsa, magnification $\times 400$ ). In panel $\mathbf{C}$ (Patient 3), there is acute chromatolysis of neurons and early infiltration by neutrophils from adjacent vessels at the edge of an area of infarction. The cerebral blood vessel (lower left) was normal and no malaria parasites were seen (H\&E, magnification $x$ 400). negative investigations for other likely pathogens, reflect the adverse and persistent neurologic effects of substantial $P$. falciparum sequestration prior to admission. The role of $P$. vivax in this process remains speculative.

There were no cases of $P$. vivax mono-infections in the present post mortem series. Children with pure severe vivax malaria in the main study had a significantly greater parasitaemia than those of $P$. vivax in the mixed infection group [2], but none of these 27 children died of cerebral malaria and only one $(3.7 \%)$ presented in deep coma (Blantyre Coma Score $\leq 2$; vs $10.3 \%$ for P. falciparum mono-infections and $22.0 \%$ for mixed species infections [2]). This is further indirect evidence against cerebral sequestration due to $P$. vivax.

The low mortality rate in the main study [2] and cultural difficulties in obtaining post mortem samples in PNG meant that there was limited availability of brain tissue. Nevertheless, detailed phenotypic, laboratory and outcome data were available for each of the three children. In addition, Plasmodium DNA was successfully extracted from brain tissue, implying that the absence of $P$. vivax DNA was not due to laboratory factors.

The present results suggest that $P$. vivax cytoadherence in brain is not a significant pathophysiologic mechanism underlying impaired consciousness in severely ill children with mixed species malaria. Other factors that underlie the poor prognosis of mixed species infections in the present series include the possibility that enhanced cytokine and/or toxin production associated with $P$. vivax [5] amplifies the effects of $P$. falciparum sequestration on vital organ function, and even that the presence of $P$. vivax in peripheral blood facilitates cytoadhesion of P. falciparum. The global burden of vivax malaria and the increasing recognition that this parasite can have adverse effects on the human host, especially in concert with P. falciparum, make this an important area for further study.

\section{Consent}

Ethical approval for the main study and present substudy was obtained from the PNG Institute of Medical Research Institutional Review Board and the Medical Research Advisory Committee of the PNG Health Department. Parent(s)/guardian(s) provided written, informed consent before a child was recruited to the main study and were subsequently asked for permission for a limited post mortem examination, including needle aspiration biopsies, if their child died.

\section{Abbreviations}

CSF: cerebrospinal fluid; H\&E: haematoxylin and eosin; nPCR: nested polymerase chain reaction; PNG: Papua New Guinea; GPCR: quantitative PCR; WHO: World Health Organization. 


\section{Acknowledgements}

The authors gratefully acknowledge the assistance of staff on the Pediatric Ward at Modilon Hospital, the Papua New Guinea Institute of Medical Research staff at Modilon Hospital and the Yagaum campus, and the patients and their families for their participation. The death of each child in this study was a tragedy and our sincere sympathies go to the families affected, but we are grateful for having received their permission to perform the post mortem brain biopsies. This study was funded by a National Health and Medical Research Council (NHMRC) grant (\#513782). The authors also acknowledge support from the MalariaGen Genomic Epidemiology Network. ML was supported by a Fogarty Foundation scholarship, LM by a Basser scholarship from the Royal Australasian College of Physicians and an NHMRC scholarship, and TMED by an NHMRC Practitioner Fellowship. None of the funding bodies were involved in study design, in the collection, analysis, and interpretation of data, in the writing of the manuscript, or in the decision to submit the manuscript for publication.

\section{Author details}

'School of Medicine and Pharmacology, University of Western Australia, Fremantle Hospital, PO Box 480, Fremantle 6959, Western Australia, Australia. ${ }^{2}$ Papua New Guinea Institute of Medical Research, Madang, Papua New Guinea. ${ }^{3}$ Department of Medicine, Faculty of Medicine Nursing and Health Sciences, Alfred Hospital, Prahran, Victoria, Australia. ${ }^{4}$ Infection and Immunity Division, Walter and Eliza Hall Institute, Parkville, Victoria, Australia. ${ }^{5}$ Centre de Recerca en Salut Internacional de Barcelona (CRESIB), Barcelona, Spain.

\section{Authors' contributions}

LM co-ordinated the main study, obtained biopsies and produced the initial draft of the paper. AR-U performed the PCR assays and edited drafts of the paper. ML assisted clinically with the main study, obtained biopsies and edited drafts of the paper. HE assisted with data collection and specimen preparation, and edited drafts of the paper. CM performed the histological examination of brain tissue and edited drafts of the paper. IM was a coinvestigator on the main study and edited drafts of the paper. PS was a coinvestigator on the main study and edited drafts of the paper. TMED was the principal investigator of the main study, conceived the sub-study and produced the final version of the paper. All authors read and approved the final manuscript.

\section{Competing interests}

The authors declare that they have no competing interests.

Received: 26 November 2011 Accepted: 3 April 2012

Published: 3 April 2012

\section{References}

1. Price RN, Tjitra E, Guerra CA, Yeung S, White NJ, Anstey NM: Vivax malaria: neglected and not benign. Am J Trop Med Hyg 2007, 77:79-87.

2. Manning L, Laman M, Law I, Bona C, Aipit S, Teine D, Warrell J, RosanasUrgell A, Lin E, Kiniboro B, Vince J, Hwaiwhanje I, Karunajeewa H, Michon P, Siba P, Mueller I, Davis TM: Features and prognosis of severe malaria caused by Plasmodium falciparum, Plasmodium vivax and mixed Plasmodium species in Papua New Guinean children. PLoS One 2011, 6: e29203.

3. Lampah DA, Yeo TW, Hardianto SO, Tjitra E, Kenangalem E, Sugiarto $P$, Price RN, Anstey NM: Coma associated with microscopy-diagnosed Plasmodium vivax: a prospective study in Papua. Indonesia. PLoS Negl Trop Dis 2011, 5:e1032.

4. Kochar DK, Tanwar GS, Khatri PC, Kochar SK, Sengar GS, Gupta A, Kochar A, Middha S, Acharya J, Saxena V, Pakalapati D, Garg S, Das A: Clinical features of children hospitalized with malaria-a study from Bikaner, northwest India. Am J Trop Med Hyg 2010, 83:981-989.

5. Anstey NM, Russell B, Yeo TW, Price RN: The pathophysiology of vivax malaria. Trends Parasitol 2009, 25:220-227.

6. Anstey NM, Handojo T, Pain MC, Kenangalem E, Tjitra E, Price RN, Maguire GP: Lung injury in vivax malaria: pathophysiological evidence for pulmonary vascular sequestration and posttreatment alveolarcapillary inflammation. J Infect Dis 2007, 195:589-596.

7. Carvalho BO, Lopes SC, Nogueira PA, Orlandi PP, Bargieri DY, Blanco YC, Mamoni R, Leite JA, Rodrigues MM, Soares IS, Oliveira TR, Wunderlich G, Lacerda MV, del Portillo HA, Araújo MO, Russell B, Suwanarusk R,
Snounou G, Rénia L, Costa FT: On the cytoadhesion of Plasmodium vivaxinfected erythrocytes. J Infect Dis 2010, 202:638-647.

8. Chotivanich K, Udomsangpetch D, Pukrittayakamee A, Looareesuwan S, Beeson J, Day N, White N: The adhesion receptors of P. vivax-infected red cells. Exp Parasitol 2003, 105:33-34.

9. Milner DA Jr, Dzamalala CP, Liomba NG, Molyneux ME, Taylor TE: Sampling of supraorbital brain tissue after death: improving on the clinical diagnosis of cerebral malaria. J Infect Dis 2005, 191:805-808.

10. Snounou G, Viriyakosol S, Jarra W, Thaithong S, Brown KN: Identification of the four human malaria parasite species in field samples by the polymerase chain reaction and detection of a high prevalence of mixed infections. Mol Biochem Parasitol 1993, 58:283-292.

11. Rosanas-Urgell A, Mueller D, Betuela I, Barnadas C, Iga J, Zimmerman PA, del Portillo HA, Siba P, Mueller I, Felger I: Comparison of diagnostic methods for the detection and quantification of the four sympatric Plasmodium species in field samples from Papua New Guinea. Malar J 2010, 9:361.

12. Davis TM, Krishna S, Looareesuwan S, Supanaranond W, Pukrittayakamee S, Attatamsoonthorn $\mathrm{K}$, White $\mathrm{NJ}$ : Erythrocyte sequestration and anemia in severe falciparum malaria. Analysis of acute changes in venous hematocrit using a simple mathematical model. J Clin Invest 1990 86:793-800.

13. Ohrt C, Sutamihardja MA, Tang D, Kain KC: Impact of microscopy error on estimates of protective efficacy in malaria-prevention trials. $J$ Infect Dis 2002, 186:540-546.

14. Pongponratn E, Turner GD, Day NP, Phu NH, Simpson JA, Stepniewska K, Mai NT, Viriyavejakul P, Looareesuwan S, Hien TT, Ferguson DJ, White NJ: An ultrastructural study of the brain in fatal Plasmodium falciparum malaria. Am J Trop Med Hyg 2003, 69:345-359.

15. Idro R, Jenkins NE, Newton CR: Pathogenesis, clinical features, and neurological outcome of cerebral malaria. Lancet Neurol 2005, 4:827-840.

doi:10.1186/1475-2875-11-107

Cite this article as: Manning et al: A histopathologic study of fatal paediatric cerebral malaria caused by mixed Plasmodium falciparum/ Plasmodium vivax infections. Malaria Journal 2012 11:107.

\section{Submit your next manuscript to BioMed Central and take full advantage of:}

- Convenient online submission

- Thorough peer review

- No space constraints or color figure charges

- Immediate publication on acceptance

- Inclusion in PubMed, CAS, Scopus and Google Scholar

- Research which is freely available for redistribution

Submit your manuscript at www.biomedcentral.com/submit
C Biomed Central 